\title{
On the degeneration of the Frölicher spectral sequence and small deformations
}

https://doi.org/10.1515/coma-2020-0003

Received April 12, 2019; accepted November 25, 2019

Abstract: We study the behavior of the degeneration at the second step of the Frölicher spectral sequence of a $e^{\infty}$ family of compact complex manifolds. Using techniques from deformation theory and adapting them to pseudo-differential operators we prove a result à la Kodaira-Spencer for the dimension of the second step of the Frölicher spectral sequence and we prove that, under a certain hypothesis, the degeneration at the second step is an open property under small deformations of the complex structure.

Keywords: Complex Geometry; Spectral Sequences; Psueudo-differential Operators

MSC: 53C56, 58C 40

\section{Introduction}

An important topic in complex geometry is the study of the behavior of certain properties under $\mathrm{C}^{\infty}$ changes, called small deformations, of the complex structure of a compact complex manifold. In one of the most celebrated papers, [10],the stability of the Kähler condition has been proved, namely if a compact complex manifold admits a Kähler metric and we change in $\mathcal{C}^{\infty}$ way its complex structure, then on the new complex manifold can be defined a Kähler metric. Moreover, as a consequence of the upper semi-continuity of the Hodge numbers with respect to the deformation of the complex structure, also the degeneration at the first step of the Frölicher spectral sequence is stable under small deformation of the complex structure. On the other way, in [1, Corollary 5.12], it has been proved that the degeneration at the second step of the Frölicher spectral sequence is not stable. In particular in Corollary 4.9 it is proved that there exists a $e^{\infty}$ family of compact complex manifolds $\left(M, J_{t}\right)$ for which the function $t \mapsto \operatorname{Dim} E_{2}^{p, q}\left(M, J_{t}\right)$ is not upper semi-continuous or lower semi-continuous. In this paper we study the stability under small deformations of the complex structure of the degeneration at the second step of the Frölicher spectral sequence. Spectral sequences are generalizations of exact sequences and they were introduced by Leray in [11] to compute the homology groups of sheaves by taking successive approximations. Given a compact complex manifold $(M, J)$, the Frölicher spectral sequence $\left(E_{r}^{\bullet, \bullet}, d_{r}\right)$ is the spectral sequence of the double complex $\left(\Lambda^{p, q}, \partial, \bar{\partial}\right)$ of $\mathcal{C}^{\infty}(p, q)$-forms of $(M, J)$ (see [5]). It provides a link between the Dolbeault and the de Rham cohomology groups. Moreover, it "measures" the failure of results in cohomology theory that are valid for Kähler manifolds (or more generally for manifolds satisfying the $\partial \bar{\partial}$-Lemma, see [3] ). The degeneration at the second step of the Frölicher spectral sequence has been studied recently in [14]; the author constructed a pseudo differential operator $\tilde{\Delta}$ whose kernel is isomorphic to $E_{2}^{\bullet, \bullet}$. Since this operator also provides relations between SKT and Gauduchon metrics and super SKT and strongly Gauduchon metrics of $(M, J)$ (see [6], [13], [7] and [12] for more information about those metrics) and since, always in [14], Popovici conjectured a connection between the degeneration at the second step of $\left(E_{r}^{\bullet, \bullet}, d_{r}\right)$ and the presence of an SKT metric on $(M, J)$, in the second section we prove some properties of $\tilde{\Delta}$.

`Corresponding Author: Michele Maschio: None Parma, Italy, E-mail: michele.maschio@student.unife.it 
As stated above, we are interested in the study of the behavior of the degeneration at the second step of $\left(E_{r}^{\bullet, \bullet}, d_{r}\right)$ on a compact complex manifold $(M, J)$ under small deformations $J_{t}$ of the complex structure $J=J_{0}$. More precisely, we prove the following

Theorem 1. Let $\left(M, J_{t}\right)$ be a family of compact complex manifolds. Suppose that, for every $(p, q) \in \mathbb{Z}^{2}, h^{p, q}(t)$ is independent of $t$ and $E_{2}^{p, q}(0) \simeq E_{\infty}^{p, q}(0)$. Then, for every $(p, q) \in \mathbb{Z}^{2}, E_{2}^{p, q}(t) \simeq E_{\infty}^{p, q}(t)$ for $t$ sufficiently close to 0 .

The basic tools in the proof of Theorem 1 are the following: the general a priori estimate

Theorem 2. For every $k \in \mathbb{Z}$ there exists a constant $C_{k}$ depending only on $k$ such that for every $\phi \in \Lambda^{p, q}(M)$

$$
\|\phi\|_{k+2}^{2} \leq C_{k}\left(\|\tilde{\Delta} \phi\|_{k}^{2}+\|\phi\|_{0}^{2}\right)
$$

and a property of self-adjoint elliptic differential operators that also holds for $\tilde{\Delta}$, namely

Theorem 3. Fix $a \mathrm{C}^{\infty}$ family $\left\{\omega_{t}\right\}$ of Hermitian metrics. If Dim Ker $\Delta_{t}^{\prime \prime}$ is independent of $t$, then Dim $\operatorname{Ker} \tilde{\Delta}_{t}$ is an upper semi-continuous function in $t$.

The paper is organized as follows:

In Section 2 we fix notations and we recall basic facts on the Frölicher spectral sequence, differential operators and Sobolev norms.

In Section 3 we recall the construction of $\tilde{\Delta}$ recently made by Popovici in [14] and we give the proof of Theorem 2.

Section 4 is devoted to the proof of Theorem 1. First of all using techniques à la Kodaira [9], we show Theorem 3 applied to the family of self-adjoint "elliptic" pseudo-differential operators $\left\{\tilde{\Delta}_{t}\right\}$. Then we use Theorems 2 and 3 to prove Theorem 1.

\section{Preliminaries}

Let $(M, J)$ be a compact complex manifold of complex dimension $n$. The main object of this paper is the Frölicher Spectral Sequence $\left(E_{r}^{p, q}, d_{r}\right)$ of $(M, J)$. Namely, denoting by $\left(\Lambda^{\bullet \bullet \bullet}(M), \partial, \bar{\partial}\right)$ the double complex of $\mathcal{C}^{\infty}$ forms over $M$, the Frölicher spectral sequence $\left(E_{r}^{p, q}, d_{r}\right)$ of $(M, J)$ is the spectral sequence associated to the complex $\left(\Lambda^{\bullet, \bullet}(M), \partial, \bar{\partial}\right)$ and it was firstly defined in [5]. We recall its construction: let $E_{0}^{p, q}:=\Lambda^{p, q}(M)$ and $d_{0}:=\bar{\partial}: \Lambda^{p, q}(M) \rightarrow \Lambda^{p, q+1}(M)$, then, inductively, let $E_{r}^{p, q}$ be the cohomology group of the $(r-1)$-th step of the sequence and $d_{r}: E_{r}^{p, q} \rightarrow E_{r}^{p+r, q-r+1}$, see, for example, [2] for detailed description of $d_{r}$. This spectral sequence satisfies the following properties:

- $E_{1}^{p, q}$ is isomorphic to the $(p, q)$-th group of the Dolbeault cohomology;

- for every $r \geq 1, E_{r}^{p, q}$ is a finite-dimensional complex vector space;

- for every $r \geq 1, \operatorname{dim} E_{r}^{p, q} \geq \operatorname{dim} E_{r+1}^{p, q}$.

Moreover the sequence is said to degenerate at the step $r$ if $r$ is the smallest integer such that, for every $(p, q) \in$ $\mathbb{Z}^{2}$ and every $r^{\prime}>r, \operatorname{dim} E_{r}^{p, q}=\operatorname{dim} E_{r^{\prime}}^{p, q}$. When this happens we have that $H_{d R}^{k}(M ; \mathbb{C}) \simeq \oplus_{p+q=k} E_{r}^{p, q}$. Since the sequence degenerates at the first step when $M$ is a Kähler manifold, we can say that it provides a "measure of the non-Kählerianity" of a manifold. In particular we are interested in the degeneration at the second step of $\left(E_{r}^{p, q}, d_{r}\right)$.

Although the spectral sequence is an algebraic object, we use analytic arguments to prove Theorem 3. This is a classic approach, in fact, for example, the Dolbeault Laplacian can be used to study the Dolbeault cohomology of a compact complex manifold. Since we will use it later on, we recall its definition: fix a Hermitian metric $g$ over $M$ and let * be the Hodge-star operator with respect to $g$, we recall that the Dolbeault 
Laplacian $\Delta^{\prime \prime}$ is defined as

$$
\Delta^{\prime \prime}:=\overline{\partial \partial}^{\star}+\bar{\partial}^{\star} \bar{\partial}
$$

where $\bar{\partial}^{\star}:=-{ }^{\star} \partial^{\star}$ is the operator adjoint to $\bar{\partial}$. It is a standard result that $\Delta^{\prime \prime}$ is an elliptic, self-adjoint and non-negative differential operator. Moreover $\Delta^{\prime \prime}$ induces the decompositions

$$
\Lambda^{p, q}(M)=\operatorname{Ker} \Delta^{\prime \prime} \oplus \operatorname{Im} \Delta^{\prime \prime}=\operatorname{Ker} \Delta^{\prime \prime} \oplus \operatorname{Im} \bar{\partial} \oplus \operatorname{Im} \bar{\partial}^{\star} .
$$

For every $(p, q)$, we denote with $\mathcal{H}^{p, q}:=\Lambda^{p, q}(M) \cap \operatorname{Ker} \Delta^{\prime \prime}$ the space of $\Delta^{\prime \prime}$-harmonic $(p, q)$ forms. It is a wellknown fact that $\mathcal{H}^{p, q}$ is a finite dimensional $\mathbb{C}$-vector space.

Finally, since we need some estimates, we recall the definition of the Sobolev norm of a $(p, q)$-form. Let $\left\{U_{j}\right\}$ be a finite covering by coordinate neighborhoods of $M$, let $\left\{x_{j}^{i}\right\}$ be coordinate over $U_{j}$ and let $\left\{\eta_{j}\right\}$ be a partition of unity subordinated to $\left\{U_{j}\right\}$. Given a $\mathcal{C}^{\infty}$ function $f$ over $M$, the $k$-th Sobolev norm of $f \in \mathcal{C}^{\infty}(M, \mathbb{C})$ is defined as

$$
\|f\|_{k}^{2}:=\sum_{|l|=0}^{k} \sum_{j} \sum_{D_{j}^{l}} \int_{U_{j}}\left|D_{j}^{l} f_{j}(x)\right|^{2} d X_{j},
$$

where

- $f_{j}:=\eta_{j} f$

- $l=\left(l_{1}, \ldots, l_{2 n}\right)$ is a multiindex and $|l|=l_{1}+\cdots+l_{2 n}$;

- $\quad D_{j}^{l}:=\frac{\partial^{l_{1}}}{\partial x_{j}^{1, l_{1}}} \cdots \frac{\partial^{l_{2 n}}}{\partial x_{j}^{2 n, l_{2 n}}}$ is a differential operator of rank $|l|$;

- $\quad d X_{j}$ is the Lebesgue measure associated to $g$ and expressed in the local coordinates $d x_{j}^{1}, \ldots, d x_{j}^{2 n}$.

If $\phi$ is an $r$-form over $M$ that, for every $U_{j}$, can be written locally as $\phi_{j}=\eta_{j} \phi=\sum_{A_{l}} f_{j}^{A_{l}} d x^{A_{l}}$ ( $d x^{A_{l}}$ stands for $d x_{l_{1}} \wedge \ldots \wedge d x_{l_{r}}$ with $\left\{l_{1}, \ldots, l_{r}\right\}=A_{l}$ and $\left.l_{1}<\ldots<l_{r}\right)$, then we define the $k$-th Sobolev norm of $\phi$ as

$$
\|\phi\|_{k}^{2}:=\sum_{j}\left\|\phi_{j}\right\|_{k}^{2},
$$

where $\left\|\phi_{j}\right\|_{k}^{2}=\sum_{A_{l}}\left\|f_{j}^{A_{l}}\right\|_{k}^{2}$

\section{The new Laplacian $\tilde{\Delta}$}

In [14], Popovici introduced a pseudo-differential operator related to the second step of the Frölicher spectral sequence in the same way the Dolbeault Laplacian is related to the first step, i.e., the Dolbeault cohomology of the manifold.

We recall its construction: let

$$
\Delta_{p^{\prime \prime}}^{\prime}:=\partial p^{\prime \prime} \partial^{\star}+\partial^{\star} p^{\prime \prime} \partial
$$

where, for every $(p, q), p^{\prime \prime}: \Lambda^{p, q}(M) \rightarrow \mathcal{H}^{p, q}$ is the orthogonal projection and $\partial^{\star}:=-\star \bar{\partial}^{\star}$ is the adjoint operator of $\partial$. Then Popovici defined the pseudo-differential operator

$$
\tilde{\Delta}:=\Delta_{p^{\prime \prime}}^{\prime}+\Delta^{\prime \prime}: \Lambda^{p, q}(M) \rightarrow \Lambda^{p, q}(M)
$$

for every $(p, q) \in \mathbb{Z}^{2} . \tilde{\Delta}$ is not a differential operator, but it is still possible to prove that it satisfies properties of elliptic operator. In particular we have

Theorem 4 ([14, Theorem 3.4]). For all $p, q, \tilde{\Delta}: \Lambda^{p, q}(M) \rightarrow \Lambda^{p, q}(M)$ behaves like an elliptic self-adjoint differential operator in the sense that Ker $\tilde{\Delta}$ is a finite dimensional $\mathbb{C}$-vector space, $\operatorname{Im} \tilde{\Delta}$ is closed and finite codimensional in $\Lambda^{p, q}(M)$, there is an orthogonal (for the $L^{2}$ inner product induced by $g$ ) 2-space decomposition

$$
\Lambda^{p, q}(M)=\operatorname{Ker} \tilde{\Delta} \oplus \operatorname{Im} \tilde{\Delta}
$$


giving rise to an orthogonal 3-space decomposition

$$
\Lambda^{p, q}(M)=\operatorname{Ker} \tilde{\Delta} \oplus\left(\operatorname{Im} \bar{\partial}+\operatorname{Im} \partial_{\operatorname{Ker} \bar{\partial}}\right) \oplus\left(\operatorname{Im} \bar{\partial}^{*}+\operatorname{Im}\left(\partial^{\star} \circ p^{\prime \prime}\right)\right)
$$

in which

$$
\begin{aligned}
& \operatorname{Ker} \tilde{\Delta} \oplus\left(\operatorname{Im} \bar{\partial}+\operatorname{Im} \partial_{\left.\right|_{\text {Ker }}}\right)=\operatorname{Ker}\left(p^{\prime \prime} \circ \partial\right) \cap \operatorname{Ker} \bar{\partial} ; \\
& \operatorname{Ker} \tilde{\Delta} \oplus\left(\operatorname{Im} \bar{\partial}^{\star}+\operatorname{Im}\left(\partial^{\star} \circ p^{\prime \prime}\right)\right)=\operatorname{Ker}\left(p^{\prime \prime} \circ \partial^{\star}\right) \cap \operatorname{Ker} \bar{\partial}^{\star} ; \\
& \left(\operatorname{Im} \bar{\partial}+\operatorname{Im} \partial_{\left.\right|_{\operatorname{Ker}} \bar{\partial}}\right) \oplus\left(\operatorname{Im} \bar{\partial}^{\star}+\operatorname{Im}\left(\partial^{\star} \circ p^{\prime \prime}\right)\right)=\operatorname{Im} \tilde{\Delta} .
\end{aligned}
$$

Moreover, $\tilde{\Delta}$ has a compact resolvent which is a pseudo-differential operator $G$ of order -2 , the Green operator of $\tilde{\Delta}$, hence the spectrum of $\tilde{\Delta}$ is discrete and consists of non-negative eigenvalues that tend to $+\infty$.

We recall that the Frölicher spectral sequence of a complex manifold $(M, J)$ is associated with the double complex $\left(\Lambda^{p, q}(M), \partial, \bar{\partial}\right)$. In particular the second step is constructed as follows: we put, for every $p, q \in \mathbb{Z}^{2}$, $E_{0}^{p, q}:=\Lambda^{p, q}(M)$ and $d_{0}:=\bar{\partial}: E_{0}^{p, q} \rightarrow E_{0}^{p, q+1}$. The groups $E_{1}^{p, q}$ at the first step in the spectral sequence are defined as the cohomology groups of the complex $\left(E_{0}^{p, q}, d_{0}\right)$, i.e. $E_{1}^{p, q}=H_{\bar{\partial}}^{p, q}(M, \mathbb{C})$ are the Dolbeault cohomology groups of $(M, J)$. The differentials $d_{1}: E_{1}^{p, q} \rightarrow E_{1}^{p+1, q}$, for every $p, q \in \mathbb{Z}^{2}$, are defined as $d_{1}\left([\alpha]_{\bar{\partial}}\right)=[\partial \alpha]_{\bar{\partial}}$. By straightforward computations we have that $d_{1}$ is well defined and that $d_{1}^{2}=0$, so $\left(E_{1}^{p, q}, d_{1}\right)$ is a complex. The groups $E_{2}^{p, q}$ at the second step in the spectral sequence are defined as the cohomology groups of the complex $\left(E_{1}^{p, q}, d_{1}\right)$, i.e.

$$
E_{2}^{p, q}:=H^{p}\left(E_{1}^{\bullet, q}, d_{1}\right)=\left\{\left[[\alpha]_{\bar{\partial}}\right] \text { s.t. } \alpha \in \Lambda^{p, q}(M) \cap \operatorname{ker} \bar{\partial} \text { and } \partial \alpha \in \operatorname{Im} \bar{\partial}\right\},
$$

for every $p, q \in \mathbb{Z}^{2}$.

In [14], Popovici defined the following two maps

$$
T=T^{p, q}: \tilde{H}^{p, q}(M) \rightarrow E_{2}^{p, q},[\tilde{\alpha}] \mapsto\left[[\alpha]_{\bar{\partial}}\right]
$$

and

$$
S=S^{p, q}: \tilde{\mathcal{H}}_{\tilde{\Delta}}^{p, q}(M) \rightarrow \tilde{H}^{p, q}(M), \alpha \mapsto[\tilde{\alpha}],
$$

where

$$
\tilde{H}^{p, q}(M):=\frac{\operatorname{Ker}\left(p^{\prime \prime} \circ \partial\right) \cap \operatorname{Ker} \bar{\partial}}{\operatorname{Im} \bar{\partial}+\operatorname{Im} \partial_{\mid \operatorname{Ker} \bar{\partial}}}
$$

and $\tilde{\mathcal{H}}_{\tilde{\Delta}}^{p, q}$ is the space of $\tilde{\Delta}$-harmonic $(p, q)$-forms. Moreover, Popovici proved that the two maps $T$ and $S$ are isomorphisms. So we have that their composition $T \circ S$ is an isomorphism between $\tilde{\mathcal{H}}_{\tilde{\Delta}}^{p, q}=\operatorname{Ker} \tilde{\Delta} \cap \Lambda^{p, q}(M, J)$ and $E_{2}^{p, q}$, for every $(p, q) \in \mathbb{Z}^{2}$.

Given the previous results, it is natural to study the behavior of $\tilde{\Delta}$ in order to better understand the Frölicher spectral sequence. In particular we want to study this behavior under small deformations of the complex structure, but we need some preliminary results.

First we study some norm estimates for $\tilde{\Delta}$. We observe that, given a $(p, q)$-form $\phi$, we have that

$$
\Delta_{p^{\prime \prime}}^{\prime} \phi=\sum_{a=1}^{l}<e_{p-1, q}^{a}, \partial^{\star} \phi>\partial e_{p-1, q}^{a}+\sum_{b=1}^{m}<e_{p+1, q}^{b}, \partial \phi>\partial^{\star} e_{p+1, q}^{b},
$$

where $l=\operatorname{Dim}_{\mathbb{C}} \mathcal{H}^{p-1, q}, m=\operatorname{Dim}_{\mathbb{C}} \mathcal{H}^{p+1, q}$ and $\left\{e_{\bullet}^{\circ}, \bullet\right\}$ is an orthonormal basis of $\mathcal{H}^{\bullet, \bullet}$ as a $\mathbb{C}$-vector space and $<\bullet, \circ\rangle$ is the standard $L^{2}$ product. Since, for every $(p, q)$-form $\psi,\left\langle e_{p, q}^{\circ}, \psi>\in \mathbb{C}\right.$ the following observation is a direct consequence of (3):

Remark 1. Locally, for every multiindex l, the derivative $D_{j}^{l}\left(\Delta_{p^{\prime \prime}}^{\prime} \phi\right)_{\mid U_{j}}$ involves only the first derivatives of $\phi$.

In the following we prove that the a priori estimate, proved for example in [9], still holds for the elliptic pseudodifferential operator with $\tilde{\Delta}$. 
Theorem 5. For every $k \in \mathbb{Z}$ there exists a constant $C_{k}$, depending on $k$, the constant of ellipticity of $\tilde{\Delta}$ and on the coefficients of $\tilde{\Delta}$, such that for every $\phi \in \Lambda^{p, q}(M)$

$$
\|\phi\|_{k+2}^{2} \leq C_{k}\left(\|\tilde{\Delta} \phi\|_{k}^{2}+\|\phi\|_{k}^{2}\right)
$$

Proof. We prove the theorem for $\mathrm{C}^{\infty}$ function, then by using (2) the general case of an arbitrary $(p, q)$-form follows straightforward. Let $\phi$ be a $e^{\infty}$ function and let $\left\{\eta_{j}\right\}$ be a partition of unity subordinated to the finite open covering $\left\{U_{j}\right\}$. Denoting with $\phi_{j}:=\eta_{j} \phi$, by the definition (1) we have

$$
\begin{aligned}
& \|\tilde{\Delta} \phi\|_{k}^{2}=\left\|\Delta^{\prime \prime} \phi+\Delta_{p^{\prime \prime}}^{\prime} \phi\right\|_{k}^{2}= \\
& \left\|\Delta^{\prime \prime} \phi\right\|_{k}^{2}+\left\|\Delta_{p^{\prime \prime}}^{\prime} \phi\right\|_{k}^{2}+\sum \int_{U_{j}}\left[\left(D_{j}^{l} \Delta^{\prime \prime} \phi_{j}\right) \overline{\left(D_{j}^{l} \Delta_{p^{\prime \prime}}^{\prime} \phi_{j}\right)}\right] d X_{j}+ \\
& +\sum \int_{U_{j}}\left[\overline{\left(D_{j}^{l} \Delta^{\prime \prime} \phi_{j}\right)}\left(D_{j}^{l} \Delta_{p^{\prime \prime}}^{\prime} \phi_{j}\right)\right] d X_{j} .
\end{aligned}
$$

Since the sum of the last two terms is a real number, it can be either negative or non-negative. If it is nonnegative, we can simply estimate

$$
\|\tilde{\Delta} \phi\|_{k}^{2} \geq\left\|\Delta^{\prime \prime} \phi\right\|_{k}^{2} .
$$

By [4], we have that there exists a constant $C_{k}$ depending only on $k$, the constant of ellipticity of $\tilde{\Delta}$ and on the coefficients of $\tilde{\Delta}$, such that

$$
\left\|\Delta^{\prime \prime} \phi\right\|_{k}^{2} \geq C_{k}^{-1}\|\phi\|_{k+2}^{2}-\|\phi\|_{k}^{2}
$$

and hence we get the conclusion.

If the sum is negative, we proceed in the following way

$$
\begin{aligned}
& \sum \int_{U_{j}}\left[\left(D_{j}^{l} \Delta^{\prime \prime} \phi_{j}\right) \overline{\left(D_{j}^{l} \Delta_{p^{\prime \prime}}^{\prime} \phi_{j}\right)}\right] d X_{j}+\sum \int_{U_{j}}\left[\overline{\left(D_{j}^{l} \Delta^{\prime \prime} \phi_{j}\right)}\left(D_{j}^{l} \Delta_{p^{\prime \prime}}^{\prime} \phi_{j}\right)\right] d X_{j}= \\
& -\left|\sum \int_{U_{j}}\left[\left(D_{j}^{l} \Delta^{\prime \prime} \phi_{j}\right) \overline{\left(D_{j}^{l} \Delta_{p^{\prime \prime}}^{\prime} \phi_{j}\right)}\right] d X_{j}+\sum \int_{U_{j}}\left[\overline{\left(D_{j}^{l} \Delta^{\prime \prime} \phi_{j}\right)}\left(D_{j}^{l} \Delta_{p^{\prime \prime}}^{\prime} \phi_{j}\right)\right] d X_{j}\right|
\end{aligned}
$$

by Stokes Theorem the second row is equal to

$$
\begin{aligned}
& -\left|\sum(-1)^{l} \int_{U_{j}}\left[\left(\phi_{j}\right)\left(\Delta^{\prime \prime} D_{j}^{l} \overline{D_{j}^{l} \Delta_{p^{\prime \prime}}^{\prime} \phi_{j}}\right)\right] d X_{j}+(-1)^{l} \int_{U_{j}}\left[\overline{\phi_{j}}\left(\overline{\Delta^{\prime \prime} D_{j}^{l}} D_{j}^{l} \Delta_{p^{\prime \prime}}^{\prime} \phi_{j}\right)\right] d X_{j}\right| \geq \\
& -\sum\left|\int_{U_{j}}\left[\phi_{j}\left(\Delta^{\prime \prime} D_{j}^{l} \overline{D_{j}^{l} \Delta_{p^{\prime \prime}}^{\prime} \phi_{j}}\right)\right] d X_{j}+\int_{U_{j}}\left[\overline{\phi_{j}}\left(\overline{\Delta^{\prime \prime} D_{j}^{l}} D_{j}^{l} \Delta_{p^{\prime \prime}}^{\prime} \phi_{j}\right)\right] d X_{j}\right| \geq \\
& -\sum \int_{U_{j}}\left|\left[\phi_{j}\left(\Delta^{\prime \prime} D_{j}^{l} \overline{D_{j}^{l} \Delta_{p^{\prime \prime}}^{\prime} \phi_{j}}\right)\right]+\left[\overline{\phi_{j}}\left(\overline{\Delta^{\prime \prime} D_{j}^{l}} D_{j}^{l} \Delta_{p^{\prime \prime}}^{\prime} \phi_{j}\right)\right]\right| d X_{j} \geq \\
& -2 \sum \int_{U_{j}}\left|\phi_{j}\left(\Delta^{\prime \prime} D_{j}^{l} \overline{D_{j}^{l} \Delta_{p^{\prime \prime}}^{\prime} \phi_{j}}\right)\right| d X_{j} \geq \\
& -2 \sum\left(\int_{U_{j}}\left|\phi_{j}\right|^{2} d X_{j}\right)^{\frac{1}{2}}\left(\int_{U_{j}} \mid\left(\left.\Delta^{\prime \prime} D_{j}^{l} \overline{D_{j}^{l} \Delta_{p^{\prime \prime}}^{\prime} \phi_{j}}\right|^{2} d X_{j}\right)^{\frac{1}{2}} \geq\right. \\
& -2 \sum\left(\int_{U_{j}}\left|\phi_{j}\right|^{2} d X_{j}\right)^{\frac{1}{2}} \sum\left(\int_{U_{j}} \mid\left(\left.\Delta^{\prime \prime} D_{j}^{l} \overline{D_{j}^{l} \Delta_{p^{\prime \prime}}^{\prime}, \phi_{j}}\right|^{2} d X_{j}\right)^{\frac{1}{2}} .\right.
\end{aligned}
$$

By Remark 1, we have that, for every $k \in \mathbb{N}$, the $k$-th Sobolev norm of $\Delta_{p^{\prime \prime}}^{\prime} \phi$ can be estimated with the 0-th norm of the first derivative of $\phi$. In fact, since $\phi$ is a $\mathcal{C}^{\infty}$ function we have that

$$
D^{l}\left(\Delta_{p^{\prime \prime}}^{\prime} \phi\right)=\sum<e_{1,0}^{a}, \partial \phi>D^{l} \partial^{\star} e_{1,0}^{a},
$$


hence

$$
\left\|\Delta_{p^{\prime \prime}}^{\prime} \phi\right\|_{k}^{2}=\sum \int_{U_{j}}\left|\sum_{a}<e_{1,0}^{a}, \partial \phi>D^{l} \partial^{\star} e_{1,0}^{a}\right|^{2} d X_{j} .
$$

Since $\left\{e_{1,0}^{a}\right\}$ is an orthonormal basis, then, for every $a=1, \ldots, \operatorname{dim} H_{\bar{\partial}}^{1,0}(M)$, there exists a constant $C_{a}^{\prime}>0$ such that $\left\langle e_{1,0}^{a}, \partial \phi\right\rangle \leq C_{a}^{\prime}\|\partial \phi\|_{0}$. Let $C^{\prime}$ be the supremum of such constant and let

$$
C_{k}^{\prime}:=\sum \int_{U_{j}}\left|D^{l} \partial^{\star} e_{1,0}^{a}\right|^{2} d X_{j},
$$

then we have

$$
\left\|\Delta_{p^{\prime \prime}}^{\prime} \phi\right\|_{k} \leq C_{k}^{\prime \prime}\left\|\phi^{\prime}\right\|_{0}
$$

where $\phi^{\prime}$ is the first derivative of $\phi$ and $C_{k}^{\prime \prime}=C_{k}^{\prime} C^{\prime}$. Thus we have

$$
-2 \sum\left(\int_{U_{j}}\left|\phi_{j}\right|^{2} d X_{j}\right)^{\frac{1}{2}} \sum\left(\int_{U_{j}} \mid\left(\left.\Delta^{\prime \prime} D_{j}^{l} \overline{D_{j}^{l} \Delta_{p^{\prime \prime}}^{\prime} \phi_{j}}\right|^{2} d X_{j}\right)^{\frac{1}{2}} \geq-2 C_{k}^{\prime \prime}\|\phi\|_{0}\left\|\phi^{\prime}\right\|_{0} \geq-C_{k}^{\prime \prime}\|\phi\|_{1}^{2} .\right.
$$

Summing up we have

$$
\|\tilde{\Delta} \phi\|_{k}^{2} \geq\left\|\Delta^{\prime \prime} \phi\right\|_{k}^{2}+\left\|\Delta_{p^{\prime \prime}}^{\prime} \phi\right\|_{k}^{2}-C_{k}^{\prime}\|\phi\|_{1}^{2}
$$

Using (4) we obtain

$$
\|\tilde{\Delta} \phi\|_{k}^{2} \geq C_{k}^{-1}\|\phi\|_{k+2}^{2}-\|\phi\|_{k}^{2}-C_{k}^{\prime}\|\phi\|_{1}^{2}
$$

If $k \geq 1$ then we have

$$
\|\tilde{\Delta} \phi\|_{k}^{2} \geq C_{k}^{-1}\|\phi\|_{k+2}^{2}-\left(1+C_{k}^{\prime}\right)\|\phi\|_{k}^{2}
$$

which is equivalent to the thesis with $C=C_{k}\left(1+C_{k}^{\prime}\right)$.

If $k=0$, we must be more precise in our estimates. In particular, by the definition of $\Delta_{p^{\prime \prime}}^{\prime}$ and denoting with $\left\{e_{1,0}^{m}\right\}$ a basis of $\mathcal{H}^{1,0}$, we have that

$$
\sum \int_{U_{j}}\left|\Delta^{\prime \prime} \Delta_{p^{\prime \prime}}^{\prime} \phi\right|^{2} d X_{j}=\sum \int_{U_{j}}\left|\Delta^{\prime \prime}\left(\sum_{m}<\partial \phi, e_{1,0}^{m}>\partial^{\star} e_{1,0}^{m}\right)\right|^{2} d X_{j} .
$$

Since $<\partial \phi, e_{1,0}^{m}>$ is a complex number, more precisely

$$
<\partial \phi, e_{1,0}^{m}>=<\phi, \partial^{\star} e_{1,0}^{m}>\leq C_{m}\|\phi\|_{0},
$$

we can estimate (5) with the 0 -th norm of $\phi$ obtaining

$$
\|\tilde{\Delta} \phi\|_{0}^{2} \geq C_{0}^{-1}\|\phi\|_{2}^{2}-\left(1+C_{0}^{\prime \prime}\right)\|\phi\|_{0}^{2},
$$

where $C_{0}^{\prime \prime}$ depends only on $\left\{e_{1,0}^{m}\right\}$.

Theorem 5 provides the basis for the estimates in the following sections. Since we need to construct the Green operator of $\tilde{\Delta}$, we prove the following

Proposition 1. If $\zeta$ is different from any eigenvalue of $\tilde{\Delta}$, then $\tilde{\Delta}-\zeta$ Id is bijective.

Proof. [9, p. 338] By construction it is obvious that $\tilde{\Delta}-\zeta I d$ is injective. We want to prove that $\tilde{\Delta}-\zeta I d$ is surjective. Let $\phi \in \mathrm{C}^{\infty}(M, \mathbb{C})$, then we can write $\phi=\sum_{j=1}^{\infty} b_{j} e_{j}$, where $\left\{e_{j}\right\}$ is an orthonormal basis of $\mathrm{C}^{\infty}(M, \mathbb{C})$ made of eigenfunctions of $\tilde{\Delta}$. By [9, Lemma 7.4], we have that, for every $l \in \mathbb{N}$,

$$
\sum_{j=1}^{\infty}\left|\lambda_{j}\right|^{2 l}\left|b_{j}\right|^{2}<\infty
$$


where $\lambda_{j}$ is the eigenvalue associated to $e_{j}$. Let $a_{j}:=b_{j} /\left(\lambda_{j}-\zeta\right)$, then

$$
\sum_{j=1}^{\infty}|\lambda|^{2 l}\left|a_{j}\right|^{2}=\sum_{j=1}^{\infty} \frac{\left|\lambda_{j}\right|^{2 l}\left|b_{j}\right|^{2}}{\left|\lambda_{j}-\zeta\right|^{2}}<\infty
$$

for every $l \in \mathbb{N}$. Thus we have that there exists $\psi \in \mathcal{C}^{\infty}(M, \mathbb{C})$ such that $\psi=\sum_{j=1}^{\infty} a_{j} e_{j}$. By direct computation we have

$$
(\tilde{\Delta}-\zeta I d) \psi=\sum_{j=1}^{\infty}\left(\lambda_{j}-\zeta\right) a_{j} e_{j}=\sum_{j=1}^{\infty} b_{j} e_{j}=\phi
$$

and hence $\tilde{\Delta}-\zeta I d$ is surjective.

\section{Small Deformations}

In this section we prove Theorem 1; to do so we use the theory of deformations of complex structures developed by Kodaira and Spencer in [10]. Our approach is similar to the one in [9] for elliptic self-adjoint differential operators and, when the proof of a statement does not change when we replace a generic differential operator with $\tilde{\Delta}$, we omit that proof.

Let $\left\{J_{t}\right\}$, with $t \in B \subset \mathbb{C}^{m}$, be a $\mathcal{C}^{\infty}$ family of complex structures over $M$ and let $\left\{\omega_{t}\right\}$ be a $\mathcal{C}^{\infty}$ family of Hermitian metrics on the fibers. Suppose that $0 \in B$. For every $t \in B$, we denote with $\Delta_{t}^{\prime \prime}, \Delta_{p^{\prime \prime} t}^{\prime}$ and $\tilde{\Delta}_{t}$ the operators described in the previous section with respect to the complex structure $J_{t}$.

Proposition 2. If Dim Ker $\Delta_{t}^{\prime \prime}$ is independent of t, then $\left\{\tilde{\Delta}_{t}\right\}$ is a $\mathrm{C}^{\infty}$ family of pseudo differential operators.

Proof. From [9], we have that all the derivative operators depend $\mathcal{C}^{\infty}$ with respect to $t$, hence we only need to prove that if $\left\{\phi_{t}\right\}$ and $\left\{\psi_{t}\right\}$ are $\mathcal{C}^{\infty}$ families of $(p, q)$ forms over $M$ and if $\left\{g_{t}\right\}$ is a $\mathcal{C}^{\infty}$ family of Hermitian metrics over $M$, then the scalar product $\left\langle\psi_{t}, \phi_{t}\right\rangle_{t}$ varies in a $C^{\infty}$ way respect to $t$. By definition

$$
\left.<\psi_{t}, \phi_{t}\right\rangle_{t}=\int_{M} \psi_{t} \wedge{ }^{\star} \bar{\phi}_{t} .
$$

Let $\left\{U_{j}\right\}$ be a finite covering of $M$ made by open coordinate neighborhoods and let $\left\{\eta_{j}\right\}$ be a partition of unity subordinate to $\left\{U_{j}\right\}$. Then, for every $t \in B$ we have

$$
\int_{M} \psi_{t} \wedge{ }^{\star} \bar{\phi}_{t}=\sum_{j} \int_{U_{j}} \eta_{j} \psi_{t} \wedge{ }^{\star}{ }_{t} \bar{\phi}_{t}
$$

Now, locally we have $\psi_{t}=\sum_{A_{p}, B_{q}} \psi_{t}^{A_{p} \bar{B}_{q}} d z^{A_{p} \bar{B}_{q}}$ and

$$
{ }^{\star} \bar{\phi}_{t}(z):=(i)^{n}(-1)^{k} \sum_{A_{p}, B_{q}} \operatorname{sgn}\left(\begin{array}{cc}
A_{p} & A_{n-p} \\
B_{q} & B_{n-q}
\end{array}\right) g_{t}(z) \bar{\phi}_{t}^{A_{p} \bar{B}_{q}}(z) d z^{B_{n-p} \overline{A_{n-q}}} .
$$

Then we have

$$
\int_{U_{j}} \eta_{j} \psi_{t} \wedge{ }^{\star} \bar{\phi}_{t}=\sum_{A_{p}, B_{q}} \sigma_{A_{p} B_{p}} \int_{U_{j}} \eta_{j} \psi_{t}^{A_{p} \bar{B}_{q}} \bar{\phi}_{t}^{A_{p} \bar{B}_{q}} g_{t} d z^{1} \wedge \cdots \wedge d z^{n} \wedge d \bar{z}^{1} \wedge \cdots \wedge d \bar{z}^{n},
$$

where $\sigma_{A_{p} B_{p}}$ is the sign of the permutation.

In order to prove that the scalar product is $\mathrm{e}^{\infty}$, it suffices to show that it is $\mathrm{e}^{k}$ for every $k \in \mathbb{N}$. We begin proving that it is $\mathcal{C}^{0}$ : by the continuity of the integral operator, the coefficients of $\psi_{t}, \phi_{t}$ and $g_{t}$ and since $\eta_{j} \psi_{t} \wedge{ }_{t}{ }_{t} \bar{\phi}$ is continuous and compactly supported in $U_{j}$, we have the continuity of the scalar product. 
Now we prove by induction over $r \in \mathbb{N}$ that (6) is $\mathcal{C}^{r}$. Let $r=1$ and consider the following

$$
\frac{\left.<\psi_{t}, \phi_{t}\right\rangle_{t}-<\psi_{s}, \phi_{s}>_{s}}{t-s}=\frac{1}{t-s} \int_{M} \psi_{t} \wedge{ }^{\star} \bar{\phi}_{t}-\psi_{s} \wedge{ }^{\star} \bar{\phi}_{s} .
$$

Locally we can rewrite the integral above as

$$
\frac{1}{t-s} \sum_{A_{p}, B_{q}} \int_{U_{j}}\left(\eta_{j} \psi_{t}^{A_{p} \bar{B}_{q}} \bar{\phi}_{t}^{A_{p} \bar{B}_{q}} g_{t}-\eta_{j} \psi_{s}^{A_{p} \bar{B}_{q}} \bar{\phi}_{s}^{A_{p} \bar{B}_{q}} g_{s}\right) d z^{1} \wedge \cdots \wedge d z^{n} \wedge d \bar{z}^{1} \wedge \cdots \wedge d \bar{z}^{n} .
$$

Now, for every multi-indexes $A_{p}$ and $B_{q}$, we consider the following construction

$$
\begin{aligned}
& \frac{1}{t-s} \eta_{j}\left(\psi_{t}^{A_{p} \bar{B}_{q}} \bar{\phi}_{t}^{A_{p} \bar{B}_{q}} g_{t}-\psi_{s}^{A_{p} \bar{B}_{q}} \bar{\phi}_{s}^{A_{p} \bar{B}_{q}} g_{s}\right)= \\
& =\frac{1}{t-s} \eta_{j}\left(\psi_{t}^{A_{p} \bar{B}_{q}} \bar{\phi}_{t}^{A_{p} \bar{B}_{q}}\left(g_{t}-g_{s}\right)+\psi_{t}^{A_{p} \bar{B}_{q}}\left(\bar{\phi}_{t}^{A_{p} \bar{B}_{q}}-\bar{\phi}_{s}^{A_{p} \bar{B}_{q}}\right) g_{s}+\left(\psi_{t}^{A_{p} \bar{B}_{q}}-\psi_{s}^{A_{p} \bar{B}_{q}}\right) \bar{\phi}_{s}^{A_{p} \bar{B}_{q}} g_{s}\right) \\
& =\eta_{j}\left(\psi_{t}^{A_{p} \bar{B}_{q}} \bar{\phi}_{t}^{A_{p} \bar{B}_{q}} \frac{g_{t}-g_{s}}{t-s}+\psi_{t}^{A_{p} \bar{B}_{q}} \frac{\bar{\phi}_{t}^{A_{p} \bar{B}_{q}}-\bar{\phi}_{s}^{A_{p} \bar{B}_{q}}}{t-s} g_{s}+\frac{\psi_{t}^{A_{p} \bar{B}_{q}}-\psi_{s}^{A_{p} \bar{B}_{q}}}{t-s} \bar{\phi}_{s}^{A_{p} \bar{B}_{q}} g_{s}\right) .
\end{aligned}
$$

When $t$ tends to $s$, we obtain that

$$
\begin{aligned}
\left.\frac{\left.d<\psi_{t}, \phi_{t}\right\rangle_{t}}{d t}\right|_{t=s}= & <\psi_{s}^{\prime}, \phi_{s}>_{s}+<\psi_{s}, \phi_{s}^{\prime}>_{s} \\
& +\sum_{j} \sum_{A_{p}, B_{q}} \int_{U_{j}} \eta_{j} \psi_{s}^{A_{p} \bar{B}_{q}} \bar{\phi}_{s}^{A_{p} \bar{B}_{q}} g_{s}^{\prime} d z^{1} \wedge \cdots \wedge d z^{n} \wedge d \bar{z}^{1} \wedge \cdots \wedge d \bar{z}^{n},
\end{aligned}
$$

where $\phi_{s}^{\prime}$ and $\psi_{s}^{\prime}$ denote the derivative along $t$ of the $\mathcal{C}^{\infty}$ forms $\phi(z, t)$ and $\psi(z, t)$ respectively. Using the same arguments of the $\mathrm{e}^{0}$ case, we have the derivative is continuous.

Suppose, by induction, that (6) is $\mathrm{e}^{r}$. By reiteration of (7) we have that the $r$-th derivative of (6) is made by two types of components

i) $<\psi_{t}^{(i)}, \phi_{t}^{(j)}>_{t}$;

ii) $\int_{M} g_{t}^{(k)} \psi_{t}^{(i)} \wedge \phi_{t}^{(j)}$, for some $k \in \mathbb{N}$.

In either case, using the same argument as above, we have the existence and the continuity of the derivative of the $r$-th derivative of $\langle\phi(z, t), \psi(z, t)\rangle t$.

Theorem 3. If Dim Ker $\Delta_{t}^{\prime \prime}$ is independent of $t$, then Dim $\operatorname{Ker} \tilde{\Delta}_{t}$ is an upper semi-continuous function in $t$.

In order to prove Theorem 3 we need some preliminary results.

Lemma 1 (Friedrichs' Inequality). For every $k \in \mathbb{N}$, there exists a constant $C_{k}$ independent of $t$ such that, for every $\mathrm{C}^{\infty}$ family $\left\{\phi_{t}\right\}$ with $\phi_{t} \in \Lambda^{p, q}\left(M, J_{t}\right)$, the inequality

$$
\left\|\phi_{t}\right\|_{k+2}^{2} \leq C_{k}\left(\left\|\tilde{\Delta}_{t} \phi_{t}\right\|_{k}^{2}+\left\|\phi_{t}\right\|_{0}^{2}\right)
$$

holds.

Proof. By Theorem 5, for every $t \in B$ and for every $k \in \mathbb{N}$ there exists a constant $C_{k, t}$ such that

$$
\left\|\phi_{t}\right\|_{k+2}^{2} \leq C_{k, t}\left(\left\|\tilde{\Delta}_{t} \phi_{t}\right\|_{k}^{2}+\left\|\phi_{t}\right\|_{k}^{2}\right)
$$

holds for every $\phi_{t} \in \Lambda^{p, q}\left(M, J_{t}\right)$. Since $\left\{g_{t}\right\}$ is a continuous family the Sobolev norm varies continuously with respect to $t$, then, up to shrinking $B$, we have that $C_{k}:=\sup \left\{C_{k, t}\right\}<\infty$. Then we obtain

$$
\left\|\phi_{t}\right\|_{k+2}^{2} \leq C_{k}\left(\left\|\tilde{\Delta}_{t} \phi_{t}\right\|_{k}^{2}+\left\|\phi_{t}\right\|_{k}^{2}\right)
$$

We will prove (8) by induction over $k$. For $k=0$, the equations (8) and (9) are the same. Then the thesis holds for $k=0$. Now suppose that thesis holds for $k-1$. By equation (8) at the step $k-1$ we have

$$
\left\|\phi_{t}\right\|_{k}^{2} \leq\left\|\phi_{t}\right\|_{k+1}^{2} \leq C_{k-1}\left(\left\|\tilde{\Delta} \phi_{t}\right\|_{k-1}^{2}+\left\|\phi_{t}\right\|_{0}^{2}\right) \text {. }
$$


Since $\left\|\tilde{\Delta} \phi_{t}\right\|_{k-1}^{2} \leq\left\|\tilde{\Delta} \phi_{t}\right\|_{k}^{2}$, we get

$$
\left\|\phi_{t}\right\|_{k+2}^{2} \leq C_{k}\left(\left\|\tilde{\Delta}_{t} \phi_{t}\right\|_{k}^{2}+\left\|\phi_{t}\right\|_{k}^{2}\right) \leq C_{k}\left(1+C_{k-1}\right)\left(\left\|\tilde{\Delta}_{t} \phi_{t}\right\|_{k}^{2}+\left\|\phi_{t}\right\|_{0}^{2}\right) .
$$

Theorem 7. If $E_{t}$ is bijective for every $t$ and if there exists a constant $c$ such that $\left\|\phi_{t}\right\|_{0} \leq c\left\|E_{t} \phi_{t}\right\|_{0}$ for every $\phi_{t} \in \Lambda^{p, q}\left(M, J_{t}\right)$. Then the family $\left\{G_{t}\right\}$ of Green operator associated to $\left\{E_{t}\right\}$ is $e^{\infty}$ differentiable in $t$.

Proof. This theorem can be proved using the same arguments of [9, Theorem 7.5] by making the following observation: by hypothesis, we have that, for every $t$

$$
\left\|\phi_{t}\right\|_{0} \leq c\left\|E_{t} \phi_{t}\right\|_{0} \leq c\left\|E_{t} \phi_{t}\right\|_{k} .
$$

Hence, there exists a constant $c^{\prime}$ independent of $t$ and $\phi_{t}$ such that

$$
\left\|\phi_{t}\right\|_{k+2} \leq c^{\prime}\left\|\tilde{\Delta}_{t} \phi_{t}\right\|_{k}
$$

By Sobolev's inequality, we have for large enough $k$ :

$$
\left|D_{j}^{l} \phi_{t j}^{\lambda}(x)\right| \leq c\left\|\phi_{t}\right\|_{k+2} .
$$

Hence

$$
\left|D_{j}^{l} \phi_{t j} j^{\lambda}(x)\right| \leq C\left\|\tilde{\Delta}_{t} \phi_{t}\right\|_{k} .
$$

Now we are in the same conditions of [9, Theorem 7.5] and we can proceed in same way.

In order to proceed we need the following result that guarantees the existence of an orthonormal basis of eigenvectors.

Lemma $2\left(\left[8\right.\right.$, Lemma 1.6.3]). Let $P: \mathcal{C}^{\infty}(M, \mathbb{C}) \rightarrow \mathcal{C}^{\infty}(M, \mathbb{C})$ be an elliptic self-adjoint pseudo-differential operator of order $d>0$. Then

- We can find a complete orthonormal basis $\left\{\psi_{n}\right\}_{n=1}^{\infty}$ for $L^{2}(M)$ of eigenvectors of $P$. $P \psi_{n}=\lambda_{n} \psi_{n}$.

- The eigenvectors $\psi_{n}$ are smooth and $\lim _{n \rightarrow \infty}\left|\lambda_{n}\right|=\infty$.

- If we order the eigenvalues $\left|\lambda_{1}\right| \leq\left|\lambda_{2}\right| \leq \ldots$ then there exists a constant $C>0$ and an exponent $\delta>0$ such that $\left|\lambda_{n}\right| \geq C n^{\delta}$ if $n>n_{0}$ is large.

Lemma 3. Let $\zeta_{0} \in \mathbb{C}$ be different from any eigenvalue of $\tilde{\Delta}_{0}$. Then there exist $\delta>0$ and $c>0$ such that, for $|t|<\delta$ and $\left|\zeta-\zeta_{0}\right|<\delta$, the following inequality

$$
\|\phi\|_{0} \leq c\left\|\tilde{\Delta}_{t}(\zeta) \phi\right\|_{0},
$$

where $\tilde{\Delta}_{t}(\zeta)=\tilde{\Delta}_{t}-\zeta$ Id, holds for every $\phi \in \Lambda^{p, q}\left(M, J_{t}\right)$.

Proof. Suppose that, for any small $\delta>0$ there is no such constant. Then, for $\mathrm{q}=1,2, \ldots$, there exist $t_{q} \in B$, $\zeta_{q} \in \mathbb{C}$ and $\phi_{q} \in \Lambda^{p, q}\left(M, J_{t}\right)$ such that

$$
\left|t_{q}\right|<\frac{1}{q}, \quad\left|\zeta_{q}-\zeta_{0}\right|<\frac{1}{q}, \quad\left\|\tilde{\Delta}_{t_{q}}\left(\zeta_{q}\right) \phi_{q}\right\|_{0}<\frac{1}{q}, \quad\left\|\phi_{q}\right\|_{0}=1 .
$$

By Lemma 1, we have

$$
\left\|\phi_{q}\right\|_{2}^{2} \leq c_{0}\left(\left\|\tilde{\Delta}_{t_{q}}\left(\zeta_{q}\right) \phi_{q}\right\|_{0}^{2}+\left\|\phi_{q}\right\|_{0}^{2}\right) \leq 2 c_{0} .
$$

Since the coefficients of $\tilde{\Delta}$ are $\mathcal{C}^{\infty}$ function on $(x, t)$ and $\tilde{\Delta}\left(\zeta_{q}\right) \phi_{q}$ is uniformly bounded, we have

$$
\left\|\tilde{\Delta}_{t_{q}}\left(\zeta_{q}\right) \phi_{q}-\tilde{\Delta}_{0}\left(\zeta_{0}\right) \phi_{q}\right\|_{0} \rightarrow 0
$$


Since $\left\|\tilde{\Delta}_{t_{q}}\left(\zeta_{q}\right) \phi_{q}\right\|_{0}<\frac{1}{q}$ then

$$
\left\|\tilde{\Delta}_{0}\left(\zeta_{0}\right) \phi_{q}\right\|_{0} \rightarrow 0
$$

But by (10) for a suitable $\mu_{0}$ we have

$$
\left\|\tilde{\Delta}_{0}\left(\zeta_{0}\right) \phi_{q}\right\|_{0} \geq \mu_{0}\left\|\phi_{q}\right\|_{0} .
$$

Hence $\left\|\phi_{q}\right\|_{0} \rightarrow 0$, which contradicts the hypothesis.

Let $C$ be a closed Jordan curve on the complex plane which does not pass through any eigenvalue of $\tilde{\Delta}_{0}$. As in [9], we denote with $((C))$ the interior of $C$. Since, for sufficient small $t, C$ does not contain any eigenvalue of $\tilde{\Delta}_{t}$, we can define the operator

$$
F_{t}(C) \phi:=\sum_{\lambda_{k}(t) \in((C))}<\phi, e_{t}^{k}>e_{t}^{k},
$$

where $\lambda_{k}(t)$ are the eigenvalue of $\tilde{\Delta}_{t}$ and $e_{t}^{k}$ is the relative eigenvector. We put $\mathbb{F}_{t}(C):=F_{t}(C) \Lambda^{p, q}\left(M, J_{t}\right)$. Obviously, $\mathbb{F}_{t}(C)$ is a finite dimensional linear subspace of $\Lambda^{p, q}\left(M, J_{t}\right)$.

Lemma 4. The operator $F_{t}(C)$ can be written as

$$
F_{t}(C) \phi=-\frac{1}{2 \pi i} \int_{C} G_{t}(\zeta) \phi d \zeta,
$$

where $G_{t}(\zeta)$ is the Green operator associated to $\tilde{\Delta}_{t}(\zeta)$.

Lemma 5. $F_{t}(C)$ is $\mathrm{e}^{\infty}$ in $t$ for $|t|<\delta$.

Proof. Since $G_{t}(\zeta)$ is $\mathcal{C}^{\infty}$ in $(t, \zeta)$, it follows that, if $\phi_{t}$ is $\mathcal{C}^{\infty}$ in $t$, then, by the definition above, also $F_{t}(C)$ is $\mathrm{e}^{\infty}$ in $t$.

Lemma 6. $\operatorname{Dim} \mathbb{F}_{t}(C)$ is independent of $t$ for $|t|<\delta$.

Proof. Let $d=\operatorname{Dim} \mathbb{F}_{0}(C)$ and let $\left\{e_{1}, \ldots, e_{d}\right\}$ be a basis for $\mathbb{F}_{0}(C)$. Since $F_{t}(C) e_{r}$ are $\mathcal{C}^{\infty}$ differentiable in $t$ and $F_{0}(C) e_{r}=e_{r}$ are linearly independent, then $F_{t}(C) e_{r}$ are linearly independent for sufficient small $t$. Hence $\operatorname{Dim} \mathbb{F}_{t} \geq d$ for sufficient small $t$.

Suppose that, for any $\delta>0$, there exists $t$ such that $|t|<\delta$ and $\operatorname{Dim} \mathbb{F}_{t}(C)>d$. Then it is possible to find a sequence $t_{q}$ such that $\left|t_{q}\right|<\frac{1}{q}$ and $\operatorname{Dim} \mathbb{F}_{t_{q}}(C)>d$. Let $\left\{\lambda_{r}^{q}\right\}$ be $d+1$ eigenvalues of $\tilde{\Delta}_{t_{q}}$ and let $\left\{e_{r}^{q}\right\}$ be the relative eigenfunctions. For a sufficient large $k$ we have

$$
\left|p_{r}^{q} D_{j}^{j} e_{r}^{q}(x)\right|^{2} \leq\left|D_{j}^{j} e_{r}^{q}(x)\right|^{2} \leq C_{k}\left(1+\sum_{\alpha=1}^{k}\left|\lambda_{r}^{q}\right|^{2}\right) .
$$

Then

$$
\left|\tilde{\Delta}_{t_{q}} e_{r}^{q}(x)\right|^{2} \leq 4 C_{k}^{2}\left(1+\sum_{\alpha=1}^{k}\left|\lambda_{r}^{q}\right|^{2}\right) .
$$

Since $\lambda_{r}^{q} \in((C))$, the sequence $\tilde{\Delta}_{t_{q}} e_{r}^{q}(x)$ is uniformly bounded in $B$. Then, up to subsequences, $\left\{\tilde{\Delta}_{t_{q}} e_{r}^{q}(x)\right\}$ converges uniformly and we have

$$
\lim _{q \rightarrow+\infty} \tilde{\Delta}_{t_{q}} e_{r}^{q}=\tilde{\Delta}_{0} e_{r}^{0}=\tilde{\Delta} e_{r} .
$$

Since $\tilde{\Delta}_{t_{q}} e_{r}^{q}=\lambda_{r}^{q} e_{r}^{q}$, the sequence $\left\{\lambda_{r}^{q}\right\}$ converges to $\lambda_{r}$ and we have

$$
\tilde{\Delta}_{0} e_{r}=\lambda_{r} e_{r} \quad\left\|e_{r}\right\|_{0}=1 .
$$

This means that $\lambda_{r}$ is an eigenvalue and $e_{r}$ an eigenfunction of $\tilde{\Delta}_{0}$. Since there are no eigenvalue on $C, \lambda_{r} \in$ $((C))$. Moreover

$$
\left(e_{r}, e_{s}\right)=\lim _{q \rightarrow+\infty}\left(e_{r}^{q}, e_{s}^{q}\right)=\delta_{r s},
$$

this means that there are $d+1$ linearly independent eigenfunctions. This contradicts the hypothesis. 
Proof of Theorem 3. To conclude the proof we only need to consider a Jordan curve passing around the eigenvalue 0 and not containing any other eigenvalue of $\tilde{\Delta}_{0}$. The thesis follows directly from Lemma 6.

Theorem 1. Let $\left(M, J_{t}\right)$ be a $\mathcal{C}^{\infty}$ family of complex manifolds and suppose that the dimension of $\operatorname{Ker} \Delta_{t}^{\prime \prime} \cap$ $\Lambda^{p, q}\left(M, J_{t}\right)$ is independent of $t$ for every $(p, q) \in \mathbb{Z}^{2}$. Then the degeneration at the second step of the Frölicher spectral sequence is stable under small deformations of the complex structure.

Proof. We denote with $b_{k}$ the dimension of $H_{d R}^{k}(M ; \mathbb{C})$, with $\tilde{h}_{t}^{p, q}$ the complex dimension of $\operatorname{Ker} \tilde{\Delta}_{t} \cap \Lambda^{p, q}\left(M, J_{t}\right)$ and with $e_{2}^{p, q}(t)$ the dimension of $E_{2}^{p, q}\left(M, J_{t}\right)$. We recall the degeneration at the second step of $\left\{E_{r}^{p, q}\left(M, J_{t}\right)\right\}$ is equivalent to

$$
b_{k}=\sum_{p+q=k} e_{2}^{p, q}(t)
$$

by Theorem [14, Theorem 3.4] we have, for every $k \in \mathbb{N}$,

$$
\sum_{p+q=k} \tilde{h}_{t}^{p, q}=\sum_{p+q=k} e_{2}^{p, q}(t)
$$

finally, by Theorem 3, we know that $\tilde{h}_{t}^{p, q}$ is an upper-semi continuous function of $t$.

Suppose that the Frölicher spectral sequence of $\left(M, J_{0}\right)$ degenerates at the second step, then, summing up all the previous considerations, we have

$$
b_{k}=\sum_{p+q=k} e_{2}^{p, q}(0)=\sum_{p+q=k} \tilde{h}_{0}^{p, q} \geq \sum_{p+q=k} \tilde{h}_{t}^{p, q}=\sum_{p+q=k} e_{2}^{p, q}(t) \geq b_{k} .
$$

Thus

$$
b_{k}=\sum_{p+q=k} \tilde{h}_{t}^{p, q}=\sum_{p+q=k} e_{2}^{p, q}(t),
$$

that means that, for $t$ small enough, the Frölicher spectral sequence of $\left(M, J_{t}\right)$ degenerates at the second step.

\section{References}

[1] M. Ceballos, A. Otal, L. Ugarte, and R. Villacampa. Invariant complex structures on 6-nilmanifolds: classification, frölicher spectral sequence and special hermitian metrics. J. Geom. Anal., 26(1):252-286, 2016.

[2] L. A. Cordero, M. Fernández, L. Ugarte, and A. Gray. A general description of the terms in the frölicher spectral sequence. Differential Geometry and its Applications, 7(1):75-84, 1997.

[3] P. Deligne, P. Griffiths, J. Morgan, and D. Sullivan. Real homotopy theory of kähler manifolds. Invent. Math., 29(3):245-274, 1975.

[4] K. O. Friedrichs. On the differentiability of the solutions of linear elliptic differential equations. Comm. Pure App. Math., 6 (3):299-326, 1953.

[5] A. Frölicher. Relations between the cohomology groups of Dolbeault and topological invariants. Proc. Natl. Acad. Sci., 41 (9):641-644, 1955.

[6] P. Gauduchon. Le théorème de l'excentricité nulle. CR Acad. Sci. Paris, 285:387-390, 1977.

[7] P. Gauduchon. La 1-forme de torsion d'une variété hermitienne compacte. Math. Ann., 267(4):495-518, 1984.

[8] P. Gilkey and D. Toledo. Invariance theory, the heat equation, and the Atiyah-Singer index theorem, volume 11. Publish or Perish Wilmington, 1984.

[9] K. Kodaira. Complex manifolds and deformation of complex structures. Springer, 2006.

[10] K. Kodaira and D. C. Spencer. On deformations of complex analytic structures, iii. stability theorems for complex structures. Ann. Math., 71:43-76, 1960.

[11] J. Leray. L'anneau spectral et l'anneau filtrè d'homologie. J. Math. Appl., 29:1-139, 1950.

[12] M. Maschio. On the cone of strong kähler with torsion metrics. Ann. Mat. Pura Appl., pages 1-13, 2017.

[13] D. Popovici. Limits of projective manifolds under holomorphic deformations. arXiv preprint arXiv:0910.2032, 2009.

[14] D. Popovici. Degeneration at $E^{2}$ of certain spectral sequences. Int. J. Math., 27(14):1650111, 2016. 